\title{
Hospitalists' Ability to Use Hand-Carried Ultrasound for Central Venous Pressure Estimation After a Brief Training Intervention: A Pilot Study
}

\author{
L. David Martin, MD¹, Roy C. Ziegelstein, MD², Eric E. Howell, MD³, Carol Martire, RDCS ${ }^{4}$, \\ David B. Hellmann, MD4, Glenn A. Hirsch, MD, MHS²*
}

\begin{abstract}
${ }^{1}$ Division of Chemical Dependence, Johns Hopkins Bayview Medical Center, Johns Hopkins University School of Medicine, Baltimore, Maryland; 2Division of Cardiology, Johns Hopkins Bayview Medical Center, Johns Hopkins University School of Medicine, Baltimore, Maryland; ${ }^{3}$ Division of Hospitalist Medicine, Johns Hopkins Bayview Medical Center, Johns Hopkins University School of Medicine, Baltimore, Maryland; ${ }^{4}$ Division of Rheumatology, Johns Hopkins Bayview Medical Center, Johns Hopkins University School of Medicine, Baltimore, Maryland.
\end{abstract}

BACKGROUND: Access to hand-carried ultrasound technology for noncardiologists has increased significantly, yet development and evaluation of training programs are limited.

OBJECTIVE: We studied a focused program to teach hospitalists image acquisition of inferior vena cava (IVC) diameter and IVC collapsibility index with interpretation of estimated central venous pressure (CVP).

METHODS: Ten hospitalists completed an online educational module prior to attending a 1-day in-person training session that included directly supervised IVC imaging on volunteer subjects. In addition to making quantitative assessments, hospitalists were also asked to visually assess whether the IVC collapsed more than 50\% during rapid inspiration or a sniff maneuver. Skills in image acquisition and interpretation were assessed immediately after training on volunteer patients and prerecorded images, and again on volunteer patients at least 6 weeks later.
RESULTS: Eight of 10 hospitalists acquired adequate IVC images and interpreted them correctly on 5 of the 5 volunteer subjects and interpreted all 10 prerecorded images correctly at the end of the 1 -day training session. At $7.4 \pm 0.7$ weeks (range, 6.9-8.6 weeks) follow-up, 9 of 10 hospitalists accurately acquired and interpreted all IVC images in 5 of 5 volunteers. Hospitalists were also able to accurately determine whether the IVC collapsibility index was more than $50 \%$ by visual assessment in 180 of 198 attempts (91\% of the time).

CONCLUSIONS: After a brief training program, hospitalists acquired adequate skills to perform and interpret handcarried ultrasound IVC images and retained these skills in the near term. Though calculation of the IVC collapsibility index is more accurate, coupling a qualitative assessment with the IVC maximum diameter measurement may be acceptable in aiding bedside estimation of CVP. Journal of Hospital Medicine 2013;8:711-714. (C) 2013 Society of Hospital Medicine
The use of hand-carried ultrasound by nonspecialists is increasing. Of particular interest to hospitalists is bedside ultrasound assessment of the inferior vena cava (IVC), which more accurately estimates left atrial pressure than does assessment of jugular venous pressure by physical examination. ${ }^{1}$ Invasively measured central venous pressure (CVP) also correlates closely with estimates from IVC imaging. ${ }^{1-4}$ Although quick, accurate bedside determination of CVP may have broad potential applications in hospital medicine, ${ }^{5-8}$ of particular interest to patients and their advocates is whether hospitalists are sufficiently skilled to perform this procedure. Lucas et al. found that 8 hospitalists trained to perform 6 cardiac assessments by hand-carried ultrasound could identify an enlarged IVC with moderate accuracy (sensitivity $56 \%$, specificity $86 \%) .{ }^{9}$ To our knowledge, no other study has examined whether hospitalists can readily develop the skills to

*Address for correspondence and reprint requests: Glenn A. Hirsch, MD, 4940 Eastern Ave., Suite 2400, Bldg. 301, Baltimore, MD 21224; Telephone: 410-550-1120; Fax: 410-550-7006; E-mail: ghirsch@jhmi.edu

Additional Supporting Information may be found in the online version of this article.

Received: June 12, 2013; Revised: September 26, 2013; Accepted: September 30, 2013

2013 Society of Hospital Medicine DOI 10.1002/jhm.2103

Published online in Wiley Online Library (Wileyonlinelibrary.com). accurately assess the IVC by ultrasound. We therefore studied whether the skills needed to acquire and interpret IVC images by ultrasound could be acquired by hospitalists after a brief training program.

\section{METHODS}

\section{Study Populations}

Hospitalists and volunteer subjects both provided informed consent to participate in this study, which was approved by the Johns Hopkins University School of Medicine Institutional Review Board. Nonpregnant volunteer subjects at least 18 years of age who agreed to attend training sessions were solicited from the investigators' ambulatory clinic patient population (see Supporting Information, Appendix A, in the online version of this article) and were compensated for their time. Volunteer subjects were solicited to represent a range of cardiac pathology. Hospitalists were solicited from among 28 members of the Johns Hopkins Bayview Medical Center's Division of Hospital Medicine, a nationally renowned academic hospitalist program comprising tenure-track faculty who dedicate at least 30\% of their time to academic endeavors.

\section{Image Acquisition and Interpretation}

A pocket-sized portable hand-carried ultrasound device was used for all IVC images (Vscan; GE Healthcare, 
Milwaukee, WI). All IVC images were acquired using the conventional methods with a subcostal view while the patient is supine. Cine loops of the IVC with respiration were captured in the longitudinal axis. Diameters were obtained approximately and by convention, approximately $2 \mathrm{~cm}$ from the IVC and right atrial junction. The IVC minimum diameter was measured during a cine loop of a patient performing a nasal sniff. The IVC collapsibility was determined by the formula: IVC Collapsibility Index $=($ IVCmax - IVCmin $/$ IVCmax), where IVCmax and IVCmin represent the maximum and minimum IVC diameters respectively. ${ }^{2}$ The IVC maximum diameters and collapsibility measurements that were used to estimate CVP are shown in the Supporting Information, Appendix B, in the online version of this article.

\section{Educational Intervention and Skills Performance Assessment}

One to 2 days prior to the in-person training session, hospitalists were provided a brief introductory online curriculum (see Supporting Information, Appendix B, in the online version of this article). Groups of 3 to 4 hospitalists then completed an in-person training and testing session ( $\sim 7$ hours total time), which consisted of a precourse survey, a didactic session, and up to 4 hours of practice time with 10 volunteer subjects supervised by an experienced board-certified cardiologist (G.A.H.) and a research echocardiography technician (C.M.). The survey included details on medical training, years in practice, prior ultrasound experience, and confidence in obtaining and interpreting IVC images. Confidence was rated on a Likert scale from $1=$ strongly confident to $5=$ not confident $(3=$ neutral $)$.

Next, each hospitalist's skills were assessed on 5 volunteer subjects selected by the cardiologist to represent a range of IVC appearance and body mass index (BMI). After appropriately identifying the IVC, they were first asked to make a visual qualitative judgement whether the IVC collapsed more than 50\% during rapid inspiration or a sniff maneuver. Then hospitalists measured IVC diameter in a longitudinal view and calculated IVC collapsibility. Performance was evaluated by an experienced cardiologist (G.A.H.), who directly observed each hospitalist acquire and interpret IVC images and judged them relative to his own hand-carried ultrasound assessments on the same subjects performed just before the hospitalists' scans. For each volunteer imaged, hospitalists had to acquire a technically adequate image of the IVC and correctly measure the inspiratory and expiratory IVC diameters. Hospitalists then had to estimate CVP by interpreting IVC diameters and collapsibility in 10 previously acquired sets of IVC video and still images. First, the hospitalists performed visual IVC collapsibility assessments (IVC collapse more than $50 \%$ ) of video clips showing IVC appearance at base- line and during a rapid inspiration or sniff, without any measurements provided. Then, using still images showing premeasured maximum and minimum IVC diameters, they estimated CVP based on calculating IVC collapsibility (see Supporting Information, Appendix B, in the online version of this article for correlation of CVP to IVC maximum diameter and collapsibility). At the end of initial training hospitalists were again surveyed on confidence and also rated level of agreement (Likert scale, $1=$ strongly agree to $5=$ strongly disagree) regarding their ability to adequately obtain and accurately interpret IVC images and measurements. The post-training survey also reviewed the training curriculum and asked hospitalists to identify potential barriers to clinical use of IVC ultrasound.

Following initial training, hospitalists were provided with a hand-carried ultrasound device and allowed to use the device for IVC imaging on their general medical inpatients; the hospitalists could access the research echocardiography technician (C.M.) for assistance if desired. The number of additional patients imaged and whether scans were assisted was recorded for the study. At least 6 weeks after initial training, the hospitalists' IVC image acquisition and interpretation skills were again assessed on 5 volunteer subjects. At the follow-up assessment, 4 of the 5 volunteers were new volunteers compared to the hospitalists' initial skills testing.

\section{Statistics}

The mean and standard deviations were used to describe continuous variables and percentages to describe proportions, and survey responses were described using medians and the interquartile ranges (25th percentile, 75 th percentile). Wilcoxon rank sum tests were used to measure the pre- and post-training differences in the individual survey responses (Stata Statistical Software: Release 12; StataCorp, College Station, TX).

\section{RESULTS}

From among 18 hospitalist volunteers, the 10 boardcertified hospitalists who could attend 1 of the scheduled training sessions were enrolled and completed the study. Hospitalists' demographic information and performance are summarized in Table 1. Hospitalists completed the initial online curriculum in an average of $18.3 \pm 7$ minutes. After the in-person training session, 8 of 10 hospitalists acquired adequate IVC images on all 5 volunteer subjects. One hospitalist obtained adequate images in 4 of 5 patients. Another hospitalist only obtained adequate images in 3 of 5 patients; a hepatic vein and the abdominal aorta were erroneously measured instead of the IVC in 1 subject each. This hospitalist later performed supervised IVC imaging on 7 additional hospital inpatients and was the only hospitalist to request additional direct 
TABLE 1. Characteristics of Hospitalists and Performance After Brief Training

\begin{tabular}{cccccc}
\hline Hospitalist & $\begin{array}{c}\text { Years in } \\
\text { Practice }\end{array}$ & $\begin{array}{c}\text { Previous Ultrasound } \\
\text { Training (Hours) }\end{array}$ & $\begin{array}{c}\text { No. of Subjects Adequately } \\
\text { Imaged and Correctly Interpreted } \\
\text { After First Session (5 Maximum) }\end{array}$ & $\begin{array}{c}\text { No. of Subjects Adequately } \\
\text { Imaged and Correctly } \\
\text { Interpreted at Follow-up (5 Maximum) }\end{array}$ & $\begin{array}{c}\text { After Study Completion Felt Training } \\
\text { Was Adequate to Perform IVC Imaging }\end{array}$ \\
\hline 1 & 5.5 & 10 & 5 & 5 & 4 \\
2 & 0.8 & 0 & 5 & 5 & 5 \\
3 & 1.8 & 4.5 & 3 & 4 & 5 \\
4 & 1.8 & 0 & 5 & 5 & 5 \\
5 & 10.5 & 6 & 5 & 5 & 5 \\
6 & 1.7 & 1 & 5 & 5 & 5 \\
7 & 0.6 & 0 & 5 & 5 & 5 \\
8 & 2.6 & 0 & 4 & 5 & 5 \\
9 & 1.7 & 0 & 5 & 5 & 5 \\
10 & 5.5 & 10 & 5 & 5 & 5 \\
\hline
\end{tabular}

NOTE: Abbreviations: IVC, inferior vena cava.

*The number of hours is a self-reported estimate, but no hospitalist had previous experience imaging the IVC.

${ }^{\dagger} 1=$ strongly disagree to $5=$ strongly agree.

supervision by the research echocardiography technician. All hospitalists were able to accurately quantify the IVC collapsibility index and estimate the CVP from all 10 prerecorded cases showing still images and video clips of the IVC. Based on IVC images, 1 of the 5 volunteers used in testing each day had a very elevated CVP, and the other 4 had CVPs ranging from low to normal. The volunteer's average BMI was overweight at 27.4, with a range from 15.4 to 37.1.

At $7.4 \pm 0.7$ weeks (range, 6.9-8.6 weeks) followup, 9 of 10 hospitalists obtained adequate IVC images in all 5 volunteer subjects and interpreted them correctly for estimating CVP. The hospitalist who performed most poorly at the initial assessment acquired adequate images and interpreted them correctly in 4 of 5 patients at follow-up. Overall, hospitalists' visual assessment of IVC collapsibility index agreed with the quantitative collapsibility index calculation in 180 of 198 (91\%) of the interpretable encounters. By the time of the follow-up assessment, hospitalists had performed IVC imaging on $3.9 \pm 3.0$ additional hospital inpatients (range, 0-11 inpatients). Lack of time assigned to the clinical service was the main barrier limiting further IVC imaging during that interval. Hospitalists also identified time constraints and need for secure yet accessible device storage as other barriers.

None of the hospitalists had previous experience imaging the IVC, and prior to training they rated their average confidence to acquire an IVC image and interpret it by the hand-carried ultrasound device at $3(3,4)$ and $3(3,4)$, respectively. After the initial training session, 9 of 10 hospitalists believed they had received adequate online and in-person training and were confident in their ability to acquire and interpret IVC images. After all training sessions the hospitalists on average rated their confidence statistically significantly better for acquiring and interpreting IVC images at $2(1,2)(P=0.005)$ and $2(1,2)$ $(P=0.004)$, respectively compared to baseline.

\section{DISCUSSION}

This study shows that after a relatively brief training intervention, hospitalists can develop and over a short term retain important skills in the acquisition and interpretation of IVC images to estimate CVP. Estimating CVP is key to the care of many patients, but cannot be done accurately by most physicians. ${ }^{10}$ Although our study has a number of limitations, the ability to estimate CVP acquired after only a brief training intervention could have important effects on patient care. Given that a dilated IVC with reduced respiratory collapsibility was found to be a statistically significant predictor of 30-day readmission for heart failure, ${ }^{11}$ key clinical outcomes to measure in future work include whether IVC ultrasound assessment can help guide diuresis, limit complications, and ultimately reduce rehospitalizations for heart failure, the most expensive diagnosis for Medicare. $^{12}$

Because hand-carried ultrasound is a point-of-care diagnostic tool, we also examined the ability of hospitalists to visually approximate the IVC collapsibility index. Hospitalists' qualitative performance (IVC collapsibility judged correctly $91 \%$ of the time without performing formal measurements) is consistent with studies involving emergency medicine physicians and suggests that CVP may be rapidly and accurately estimated in most instances. ${ }^{13}$ There may be, however, value to formally measuring the IVC maximum diameter, because it may be inaccurately visually estimated due to changes in scale when the imaging depth is adjusted. Accurately measuring the IVC maximum diameter is important because a maximum diameter of more than $2.0 \mathrm{~cm}$ is evidence of an elevated right atrial pressure $(82 \%$ sensitivity and $84 \%$ specificity for predicting right atrial pressure of $10 \mathrm{~mm} \mathrm{Hg}$ or above) and an elevated pulmonary capillary wedge pressure $(75 \%$ sensitivity and $83 \%$ specificity for pulmonary capillary wedge pressure of $15 \mathrm{~mm} \mathrm{Hg}$ or more). ${ }^{14}$ 


\section{Limitations}

Our findings should be interpreted cautiously given the relatively small number of hospitalists and subjects used for hand-carried ultrasound imaging. Although our direct observations of hospitalist performance in IVC imaging were based on objective measurements performed and interpreted accurately, we did not record the images, which would have allowed separate analyses of inter-rater reliability measures. The majority of volunteer subjects were chronically ill, but they were nonetheless stable outpatients and may have been easier to position and image relative to acutely ill inpatients. Hospitalist self-selected participation may introduce a bias favoring hospitalists interested in learning hand-carried ultrasound skills; however, nearly half of the hospitalist group volunteered and enrollments in the study were based only on their availability for the previously scheduled study dates.

\section{IMPLICATIONS FOR TRAINING}

Our study, especially the assessment of the hospitalists' ability to retain their skills, adds to what is known about training hospitalists in hand-carried ultrasound and may help inform deliberations among hospitalists as to whether to join other professional societies in defining specialty-specific bedside ultrasound indications and training protocols., ${ }^{9,15}$ As individuals acquire new skills at variable rates, training cannot be defined by the number of procedures performed, but rather by the need to provide objective evidence of acquired procedural skills. Thus, going forward there is also a need to develop and validate tools for assessment of competence in IVC imaging skills.

Disclosures: This project was funded as an investigator-sponsored research project by General Electric (GE) Medical Systems Ultrasound and Primary Care Diagnostics, LLC. The devices used in this training were supplied by GE. All authors had access to the data and contributed to the preparation of the manuscript. GE was not involved in the study design, analysis, or preparation of the manuscript. All authors received research support to perform this study from the funding source.

\section{References}

1. Brennan JM, Blair JE, Goonewardena S, et al. A comparison by medicine residents of physical examination versus hand-carried ultrasound for estimation of right atrial pressure. Am J Cardiol. 2007;99(11): 1614-1616.

2. Kircher BJ, Himelman RB, Schiller NB. Noninvasive estimation of right atrial pressure from the inspiratory collapse of the inferior vena cava. Am J Cardiol. 1990;66:493-496.

3. Brennan JM, Blair JE, Goonewardena S, et al. Reappraisal of the use of inferior vena cava for estimating right atrial pressure. J Am Soc Echocardiogr. 2007;20:857-861.

4. Goonewardena SN, Blair JE, Manuchehry A, et al. Use of handcarried ultrasound, B-type natriuretic peptide, and clinical assessment in identifying abnormal left ventricular filling pressures in patients referred for right heart catheterization. J Cardiac Fail. 2010;16:69-75.

5. Blehar DJ, Dickman E, Gaspari R. Identification of congestive heart failure via respiratory variation of inferior vena cava diameter. Am J Emerg Med. 2009;27:71-75.

6. Dipti A, Soucy Z, Surana A, Chandra S. Role of inferior vena cava diameter in assessment of volume status: a meta-analysis. Am J Emerg Med. 2012;30(8):1414-1419.e1.

7. Ferrada P, Anand RJ, Whelan J, et al. Qualitative assessment of the inferior vena cava: useful tool for the evaluation of fluid status in critically ill patients. Am Surg. 2012;78(4):468-470.

8. Guiotto G, Masarone M, Paladino F, et al. Inferior vena cava collapsibility to guide fluid removal in slow continuous ultrafiltration: a pilot study. Intensive Care Med 2010;36:692-696.

9. Lucas BP, Candotti C, Margeta B, et al. Diagnostic accuracy of hospitalist-performed hand-carried ultrasound echocardiography after a brief training program. J Hosp Med. 2009;4(6):340-349.

10. Badgett RG, Lucey CR, Mulrow CD. Can the clinical examination diagnose left-sided heart failure in adults? JAMA. 1997;277: $1712-1719$.

11. Goonewardena SN, Gemignani A, Ronan A, et al. Comparison of hand-carried ultrasound assessment of the inferior vena cava and $\mathrm{N}$-terminal pro-brain natriuretic peptide for predicting readmission after hospitalization for acute decompensated heart failure. JACC Cardiovasc Imaging. 2008;1:595-601.

12. Jencks SF, Williams MV, Coleman EA. Rehospitalizations among patients in the Medicare Fee-for-Service Program. N Engl J Med. 2009;360:1418-1428.

13. Fields JM, Lee PA, Jenq KY, et al. The interrater reliability of inferior vena cava ultrasound by bedside clinician sonographers in emergency department patients. Acad Emerg Med. 2011;18:98-101.

14. Blair JE, Brennan JM, Goonewardena SN, et al. Usefulness of handcarried ultrasound to predict elevated left ventricular filling pressure. Am J Cardiol. 2009;103:246-247.

15. Martin LD, Howell EE, Ziegelstein RC, et al. Hospitalist performance of cardiac hand-carried ultrasound after focused training. Am J Med. 2007;120(11):1000-1004. 